\title{
The Anelasticity of the Earth ${ }^{1}$
}

\author{
Don L. Anderson and C. B. Archambeau \\ Seismological Laboratory \\ California Institute of Technology, Pasadena
}

\begin{abstract}
The attenuation of seismic waves is one manifestation of the earth's anelasticity and is not unrelated to the response of the earth to stresses of longer duration. The wellknown difficulties involved in the extraction of meaningful amplitude information from body waves have prevented an accurate determination of attenuation of seismic energy versus depth. Most of these difficulties are not present in surface wave and free oscillation measurements, but there are complexities of interpretation. A method is developed for the analysis of the amplitudes of dispersed wave trains and free oscillations which yields the anelasticity $(Q)$ as a function of depth in the earth just as the frequency spectrum yields the elasticity-density structure. The advantages and limitations of the method are essentially identical to those of the dispersion method. The amplitude decay versus period for toroidal oseillations and Love waves was computed for a variety of hypothetical $Q$ distributions in the earth. Those models which satisfy the available attenuation measurements have a broad, highly attenuating zone in the upper mantle and a high-Q lower mantle. The range of $Q$ for shear waves in these models is from about 80 in the upper mantle to about 2000 in the lower mantle. A rapid increase in $Q$ beginning at about $400 \mathrm{~km}$ seems to be a required feature. This is probably the most direct evidence for inhomogeneity, possibly a phase change, beginning at this depth. The details of this transition zone must await more accurate data on surface wave attenuation. The high $Q$ of the lower mantle seems to imply temperatures substantially below the melting point, and it probably precludes extensive lower mantle convection. There is no need to invoke a frequency-dependent $Q$ in order to satisfy available body and surface wave data in the period range 10 seconds to 30 minutes, although a $Q$ that is frequency dependent cannot be ruled out.
\end{abstract}

Introduction. The attenuation of seismic energy in the earth, being a direct measure of anelasticity, is potentially an important source of information regarding the composition, state, and temperature of the deep interior. The classical seismological approach is to measure and correlate amplitudes of body waves from place to place on the earth's surface, a notoriously difficult task involving corrections for source, instrument, and path. Body waves are subject to scattering, reflection, and refraction, and, since measurements are restricted to the surface of the earth, the decrease in amplitude along a given ray generally cannot be measured. The presence of a low-velocity region also complicates the extraction of useful amplitude information from the affected rays. To date, very little has been learned about the absorption properties as a function of depth in the earth.

The attenuation of long-period waves and the

1 Contribution 1236, Division of Geological Sciences, California Institute of Technology, Pasadena. decay of the free oscillations of the earth depend in a very complicated way on the distribution of parameters of elasticity and anelasticity. However, complications of source, instrument, and path differences are minimized in studies of this sort. Because of the long wavelengths of these vibrations, such effects as scattering and reflection are reduced, and we are more likely to be measuring an intrinsic property of the material. The complications due to discontinuities or low-velocity zones are also minimized.

In this paper the formal theory of surface wave attenuation and free oscillation dccay for a dissipative earth is derived. A preliminary determination is made of the distribution of $Q$ with depth.

The intrinsic dissipation of a homogeneous isotropic material is unambiguously determined by the time rate of decay of the energy in a normal mode or the spatial rate of decay of a propagating nondispersive pure mode. A useful measure of attenuation is the dimensionless quality factor $Q$, whose inverse is the specific dissipation function, 


$$
Q^{-1}=\Delta \mathrm{H} / 2 \pi V_{\max }
$$

where $\Delta \mathbf{H}$ is the strain energy dissipated per cycle and $V_{\max }$ is the maximum strain energy stored during the cycle. If the exponential time rate of amplitude attenuation is $\omega^{*}$,

$$
Q^{-1}=2 \omega^{*} / \omega
$$

where $\omega$ and $\omega^{*}$ are, respectively, the real and imaginary parts of the angular frequency. In cerms of the exponential spatial attenuation coefficient

$$
Q^{-1}=2 k^{*} / k=2 k^{*} v / \omega
$$

where $k$ and $k^{*}$ are, respectively, the real and imaginary parts of the wave number corresponding to a mode of frequency $\omega$ propagating with an intrinsic velocity $v$.

Bounded mediums, such as rods and plates, or layered mediums such as the earth, are dispersive, and the analysis is less straightforward.

A layered medium, for example, supports surface waves which propagate with a phase velocity $c=\omega / k$ and a group velocity $U=\partial \omega / \partial k$, both of which are complicated functions of the layer densities as well as the intrinsic body wave velocities of each layer. The calculation of $c$ and $U$ for arbitrarily layered half-spaces and spheres is the subject of a vast geophysical literature. The observed attenuation of surface waves is presumably an even more complicated function of the intrinsic dissipative properties of each layer, as well as the densities and elastic properties. The straightforward but tedious approach would be to assume a rheological law which is introduced into the equations of motion and boundary conditions and to solve the resulting complex boundary value problem. The simplest such rheological law results from introducing complex moduli of elasticity into Hooke's law. We will demonstrate a simpler method which makes it possible to utilize information obtained from the solution of the nondissipation problem.

First let us consider the definition of the dissipation function for a layered medium at pe$\operatorname{riod} T$ :

$$
\begin{aligned}
Q^{-1}(T)=\sum_{i} \Delta \mathrm{H}_{i}(T) / 2 \pi \sum_{i} V_{i} \\
=\sum_{i}\left(V_{i} / V\right) Q_{i}^{-1}(T)
\end{aligned}
$$

where the summation is over all of the layers ( $i$ is the layer index) contributing to the observed attenuation and $V$ is the maximum potential energy stored in the whole system during a cycle. The contribution of each layer, or shell, to the total attenuation at period $T$ is proportional to the fractional strain energy stored in each layer at that period. The $Q_{i}$ are the individual layer dissipation functions, which may be frequency dependent.

The above definitions, equations 1 and 2 , imply that all losses are due to departures from ideal elasticity. However, other mechanisms may contribute to the observed dissipation. Let us therefore define an observed specific dissipation functions, $Q_{0}{ }^{-1}$, which is identical to the above definitions, with the modifying words elastic and potential removed. Experimental data are conveniently discussed in terms of $Q_{0}{ }^{-1}$, but these discussions cannot reveal anything about the details of the dissipation mechanism. For example, the experimental facts describing the propagation of a shear wave are the shear velocity $\beta$ and the spatial attenuation coefficient $k_{\beta}{ }^{*}$. In terms of intrinsic physical properties of the material,

$$
\begin{aligned}
\beta^{2} & =\left(\omega / k_{\beta}\right)^{2}=\mu / \rho \\
k_{\beta}^{*} / k_{\beta} & =\frac{1}{2}\left[\left(\mu^{*} / \mu\right)-\left(\rho^{*} / \rho\right)\right]
\end{aligned}
$$

showing that we cannot recover the intrinsic properties $\mu, \mu^{*}, \rho$, and $\rho^{*}$ from characteristics of the propagating wave. To obtain $\mu$ and $\mu^{*}$ we must assume knowledge of $\rho$ and $\rho^{*}$.

We will show that having the $Q^{\prime}$ s weighted according to fractional elastic or potential energies is equivalent to having complex coefficients of elasticity, or imperfect elasticity. Imperfect inertia, on the other hand, is equivalent to complex densities, and the energy loss in each layer is proportional to the fractional kinetic energy in the layer.

Basic theory. The basic ideas associated with the present approach are, in nearly every respect, identical to those appropriate to the interpretation of the phase or frequency spectrums. Under certain assumptions the phase spectrum yields the velocity-density structure of the earth; the amplitude spectrum yields the dissipation, or $Q$, structure of the earth. These structures can be systematically obtained by means of a perturbation theory which is basically 
an extension of Rayleigh's principle [see for example Jeffreys, 1961; Meissner, 1926].

An outline of the theory was presented earlier by Archambeau and Anderson [1963]. Detailed applications and an alternative formulation of this method, in which Rayleigh's principle was used explicitly, have also been given by Anderson [1963, 1964]. Preliminary conclusions regarding the variation of $Q$ with depth were given by Anderson [1963] and Anderson et al. [1963]. A comprehensive theory of inversion, incorporating a general perturbation method similar to that described here, including a study of the discrete layer approximation and uniqueness, is in preparation. That part of the general theory appropriate to dissipation problems will be presented in the following theoretical discussion.

To make clear its full powers and generality, it is perhaps best to introduce the theory in an operational form wherein it takes on an especially symmetric and rather concise form. It will immediately be assumed, without loss of generality, that the medium in question is a layered structure. The interesting problems associated with the discrete layer approximation to a continuously varying structure are purely academic and outside the scope of the present study.

Let $H_{s}$ denote differential operations of the form

$$
\begin{array}{r}
H_{s} \equiv P_{1}^{s}\left[\frac{d}{d x}\left(r_{1}(x) \frac{d}{d x}\right)+P_{3}\right] \\
s=1,2, \cdots, N
\end{array}
$$

where the $P_{1}$ are real or complex constants and $N$ is the total number of layers or shells in the system. The parameters $P_{s}^{*}$ will hereafter be specialized to the single parameter $P_{3}$, the wave number, which is independent of $s$. This differential expression is of sufficient generality to serve in the investigation of any type of isotropic elastic wave propagation and for some types of anisotropic propagation as well. In most cases the eigenfunctions associated with this operation will correspond to a potential of one sort or another; for Love waves or toroidial oscillations they will represent displacements in the medium. This latter case is the subject of the experimental observations reported in this study and will therefore be emphasized in the following theoretical develop- ments. However, by redefining energies wherever they appear so as to be proper for the wave type or else by omitting energy definitions entirely, the theory presented can be applied directly to similar observations for Rayleigh waves or spheroidal oscillations in a layered earth with $N / 2$ layers. (In this case there are $N$ operators of the type in (3), grouped two to a layer, since two potentials are necessary to describe the motion. The number $N$ may of course be chosen to be even.)

The boundary value problem appropriate to the general case has the form

$$
\begin{aligned}
H_{s} u_{l}{ }^{s}= & \lambda_{l} P_{2}{ }^{s} r_{2} u_{l}{ }^{s} \\
& s=1,2, \cdots, N \\
L_{s+1} u_{l}{ }^{s+1}= & L_{s} u_{l}{ }^{s} \\
& s=1,2, \cdots, N-1 \\
M_{s+1} u_{l}{ }^{s+1}= & M_{s} u_{l}{ }^{s} \\
& s=1,2, \cdots, N-1 \\
R_{N} u_{l}{ }^{N}= & R_{1} u_{l}{ }^{1}=0
\end{aligned}
$$

The first of these equations corresponds to the equations of motion appropriate to a given layer, where $\lambda_{l}$ and $u_{l}{ }^{2}$ are the eigenvalues and eigenfunctions obtained by satisfying the stated boundary conditions. These conditions correspond to the continuity of stress and displacement at the layer interfaces and vanishing of stress at the free surface. Also, a condition at the other extremity of the range of the variable $x$ is always required: either the eigenfunctions must be finite (e.g., a radiation or Sommerfeld condition) or the stress must vanish (Love waves or toroidal oscillations in a spherical earth with a nonsolid core). The lower equations in (4) express, symbolically, these conditions at the extremities of the range of $x$. The relations involving $M_{s}$ and $L_{s}$, defined as operators of differential type, represent the continuity of displacement and stress, respectively. The boundary operators take on different forms, depending upon the wave type under consideration. They are, however, such that when the parameters $P_{i}$ : are all real the operators $H_{s}$ are a self-adjoint system. In this case the eigenvalues $\lambda_{l}$ are real.

The parameter $P_{2}$ may be real or complex. This parameter and the associated function $r_{2}(x)$ may be incorporated in the differential operator, 
but it will be convenient to consider their explicit influence in subsequent developments.

In the present context the domain of an operator $H$, is defined to include all those functions for which the operation $H_{8} u$ is meaningful and which are square-integrable over the range $x_{s-1}<x<x_{s}$, such a condition being denoted by $u \in L^{2}\left(x_{s-1}, x_{s}\right)$. Also, the first and second derivatives of functions in the domain of $H$, are defined to be in $L^{2}\left(x_{s-1}, x_{s}\right)$. Finally the domain of $H_{s}$, denoted $\mathfrak{D}\left(H_{s}\right)$, is defined to include only those functions satisfying the boundary conditions of (4). Thus the complete boundary value problem, including conditions of continuity and integrability, can be stated as

$$
\begin{aligned}
H_{s} u_{l}{ }^{s} & =\lambda_{l} P_{2}{ }^{s} r_{2} u_{l}{ }^{s} \\
u_{l}{ }^{s} & \in D\left(H_{s}\right)
\end{aligned}
$$

In physical problems the integrability of the functions $u_{i}^{*}$ and their derivatives implies the existence of kinetic and potential energies and a dissipation function when certain of the $P_{i}$ * are complex.

Clearly, the inner product defined by

$$
\{u, v\}=\int_{x_{x-1}}^{x_{*}} u \tilde{v} d x
$$

exists for all functions $u_{i}^{*}$ and $v_{i}^{*}$ in $\mathscr{D}\left(H_{s}\right)$. On this basis it is clear, then, that

$$
\lambda_{l}=\sum_{s=1}^{N}\left\{H_{s} u_{l}^{s}, u_{l}^{s}\right\} / \sum_{s=1}^{N}\left\{P_{2}^{s} r_{2} u_{l}{ }^{s}, u_{l}^{s}\right\}
$$

whenever the $u_{i}$ satisfy (4).

If the operators $H_{s}$ defined in (3) through (5) are appropriate for elastic wave motion in a dissipationless medium, the eigenvalues $\lambda_{l}$ are real, the $H_{s}$ are a self-adjoint system, and the coefficients $P_{i}$ are all real constants. For such a nondissipative physical problem, the potential energy $V_{\mathfrak{l}}$ and kinetic energy $T_{\mathfrak{l}}$ are given by

$$
\begin{aligned}
& 2 V_{l}=\sum_{s=1}^{N}\left\{H_{s} u_{l}^{s}, u_{l}^{s}\right\} \\
& 2 T_{l}=\lambda_{l} \sum_{s=1}^{N}\left\{P_{2}^{s} r_{2} u_{l}^{s}, u_{l}{ }^{s}\right\}
\end{aligned}
$$

Equation 6 therefore states that the kinetic and potential energies are equal when $u_{t}^{*}$ and $\lambda_{\imath}$ are proper values, that is, at resonance.
The problem of actual interest is the following. Let

$$
\mathfrak{H}_{s}=\bar{P}_{1}^{s}\left[\frac{d}{d x}\left(r_{1}(x) \frac{d}{d x}\right)+\bar{P}_{3}\right]
$$

where the $\bar{P}_{i}$ are complex. Then consider the system of equations

$$
\begin{aligned}
\mathscr{H}_{s} U_{l}{ }^{s} & =\Lambda_{l} \bar{P}_{2}{ }^{s} r_{2} U_{l}{ }^{s} \quad s=1,2, \cdots, N \\
U_{l}{ }^{s} & \in \mathscr{D}\left(\mathfrak{H C}_{s}\right)
\end{aligned}
$$

such that the domain of $\mathcal{H}_{\mathrm{a}}$ is defined by the same conditions as for $D\left(H_{s}\right)$, except that now at least some of the coefficients $\bar{P}_{i}^{*}$ appearing in these conditions are complex. This system therefore corresponds to wave motion in a dissipative medium, and the eigenvalues $\Lambda_{l}$ are complex.

The eigenvalue problem for the nondissipative medium will be taken as an auxiliary problem and the coefficients $P_{i}$ * will be chosen as the real parts of the complex parameters $\bar{P}_{i}$. Thus let

$$
\begin{aligned}
\delta P_{i}^{*} & =\bar{P}_{i}^{*}-P_{i}^{*} \\
\delta P_{3} & =\bar{P}_{3}-P_{3} \\
\delta \lambda_{l} & =\Lambda_{l}-\lambda_{l}
\end{aligned}
$$

and consider the relations

$$
\begin{gathered}
\sum_{s=1}^{N}\left\{H_{s} u_{l}^{s}, u_{l}{ }^{s}\right\}=\lambda_{l} \sum_{s=1}^{N}\left\{P_{2}^{s} r_{2} u_{l}{ }^{s}, u_{l}{ }^{s}\right\} \\
\sum_{s=1}^{N}\left\{\mathcal{K}_{s} U_{l}^{s}, U_{l}^{s}\right\}=\Lambda_{l} \sum_{s=1}^{N}\left\{\bar{P}_{2}^{s} r_{2} U_{l}^{s}, U_{l}{ }^{s}\right\}
\end{gathered}
$$

under the condition that the dissipation is a small effect, i.e., $\delta \lambda_{\imath}$ and $\delta P_{\imath}$ ' are small. Here a perturbation theory is clearly applicable. From the definitions of $\mathcal{H}_{s}$ and $H_{s}$, to first order,

where

$$
\mathcal{H C}_{s} \equiv H_{s}+\delta P_{1}{ }^{s} H_{1}{ }^{s}+\delta P_{3} H_{3}{ }^{s}
$$

$$
H_{1}{ }^{s} \equiv \frac{d}{d x}\left(r_{1}(x) \frac{d}{d x}\right)+P_{3} \quad H_{3}{ }^{s} \equiv P_{1}{ }^{s}
$$

Also, $U_{\boldsymbol{l}}$ may be expanded as

$$
\begin{aligned}
U_{l}{ }^{s} & =u_{l}{ }^{s}+\sum_{k, i}\left(\frac{\partial U_{l}{ }^{s}}{\partial \vec{P}_{i}{ }^{k}}\right)_{P_{i} k} \delta P_{i}{ }^{k} \\
& +\left(\frac{\partial U_{l}^{s}}{\partial \Lambda}\right)_{\lambda_{l}} \delta \lambda_{l}+O\left(\delta P_{i}{ }^{2}, \delta \lambda_{l}{ }^{2}\right)
\end{aligned}
$$


Introducing (11) and (12) in the second of equations 10 and using the self-adjoint properties of the $H_{s}$, so that

$$
\sum_{s=1}^{N}\left\{H_{s} v_{l}^{s}, u_{l}{ }^{s}\right\}=\sum_{s=1}^{N}\left\{v_{l}{ }^{s}, H_{s} u_{l}{ }^{s}\right\}
$$

and using the fact that $H_{s} u_{l}{ }^{*}=\lambda_{\imath} u_{l}{ }^{*}$ for $\lambda_{l}$ real, we have, to first order, density structure of the medium has been determined from dispersion data, for example, all the required parameters, including the eigenvalues for the auxiliary problem, will have been determined.

The results may be put in yet another form which is indicative of the nature of the dissipational processes. First, observing that $P_{1}^{2} H_{1}^{\prime}=$

$$
\delta \lambda_{l}=\frac{\sum_{s=1}^{N} \sum_{i=1}^{2}\left\{H_{i}{ }^{s} u_{l}{ }^{s}, u_{l}{ }^{s}\right\} \delta P_{i}{ }^{s}+\delta P_{3}\left\{H_{3}{ }^{s} u_{l}{ }^{s}, u_{l}{ }^{s}\right\}}{\sum_{s=1}^{N}\left\{P_{2}{ }^{s} r_{2} u_{l}{ }^{s}, u_{l}{ }^{s}\right\}}
$$

where

$$
\begin{aligned}
H_{1}{ }^{s} \equiv \frac{d}{d x}\left(r_{1}(x) \frac{d}{d x}\right) & +P_{3} \\
H_{2}{ }^{s} & \equiv-r_{2}(x) \quad H_{3}{ }^{s} \equiv P_{1}{ }^{s}
\end{aligned}
$$

This expression therefore shows the effect of dissipation on the eigenvalue. It is clear that the first-order effect is to introduce a purely imaginary part to the eigenvalue since the $\delta P_{i}^{*}$ are all imaginary. Changes in the real part of the eigenvalue are therefore of second order.

It is convenient to express this result in a form analogous to partial derivatives. Thus, we consider the ratio of the variation in the eigenvalue to the variation or perturbation of one of the parameters (corresponding to the introduction of dissipation) while all the other parameter variations or complex parts are zero. This procedure gives, for $i=1,2$ and $\delta P_{3}=0$,

$$
\left(\frac{\delta \lambda_{l}}{\delta P_{i}^{s}}\right)_{0}=\left\{H_{i}^{s} u_{l}^{s}, u_{l}^{s}\right\} / \sum_{s=1}^{N}\left\{P_{2}^{s} r_{2} u_{l}^{s}, u_{l}^{s}\right\}
$$

where $\bar{P}_{j}{ }^{*}=P_{j}^{*}$ for all $r, j$ and $j \neq i$ when $r=s$. For $\bar{P}_{i}=P_{i}$ for all $i$ and $s$,

$$
\begin{aligned}
& \left(\frac{\delta \lambda_{l}}{\delta P_{s}}\right)_{0} \\
& =\sum_{s=1}^{N}\left\{P_{1}^{s} u_{l}^{s}, u_{l}^{s}\right\} / \sum_{s=1}^{N}\left\{P_{2}^{s} r_{2} u_{l}^{s}, u_{l}^{s}\right\}
\end{aligned}
$$

These partial variations are expressed in terms of the eigenfunctions $u_{i}$ and operators $H_{i}{ }^{*}$ for the nondissipative medium and can be obtained if the real parts of the parameters $\bar{P}_{i}^{*}$ are known. In a practical case in which the velocity-
$H_{8}$ and that the potential and kinetic energies are given by (7), we see that (14) gives

$$
\begin{aligned}
\frac{P_{1}^{s}}{\lambda_{l}}\left(\frac{\delta \lambda_{l}}{\delta P_{1}^{s}}\right)_{0} & =2\left(\frac{V_{s}}{E}\right)_{l} \\
& \frac{P_{2}^{s}}{\lambda_{l}}\left(\frac{\delta \lambda_{l}}{\delta P_{2}^{s}}\right)_{0}=-2\left(\frac{T_{s}}{E}\right)_{l}
\end{aligned}
$$

where $E_{l}$ is the total energy and $V_{s}$ and $T_{s}$ are energies within the sth layer. These expressions imply that the dissipation introduced by $P_{1}^{\prime}$ is associated with the potential energy of the layer and hence is due to anelastic processes. Thus this factor is a measure of the elastic energy converted into heat or into permanent deformation work by any of the anelastic processes, including creep. This term therefore depends on the relative displacements between particles, or the elastic deformation, and vanishes in the absence of relative displacement. The term involving $P_{z}$ is associated with the kinetic energy in the sth layer and can only represent an imperfect inertial effect since it depends on the absolute velocities of the particles. It is also interesting to note that this term has a negative sign. This has at least two implications. First, since $\delta \lambda_{t}$ must have the same sign, whether it arises from either $\bar{P}_{1}^{*}$ or $\bar{P}_{2}^{*}$, it is clear that $\delta P_{2}^{*}$ must have the opposite sign to that of $\delta P_{1}^{*}$ for compliance with causality and the second law of thermodynamics. Second, the total change in $\delta \lambda_{i}$ due to both effects occurring simultaneously is given by

$$
\begin{aligned}
\frac{\delta \lambda_{l}}{\lambda_{l}}= & \sum_{s=1}^{N}\left[\left(\frac{\delta \lambda_{l}}{\delta P_{1}^{s}}\right)_{0} \frac{\delta P_{1}^{s}}{\lambda_{l}}+\left(\frac{\delta \lambda_{l}}{\delta P_{2}^{s}}\right)_{0} \frac{\delta P_{2}^{s}}{\lambda_{l}}\right] \\
& =2 \sum_{s=1}^{N}\left[\left(\frac{V_{s}}{E}\right)_{l} \frac{\delta P_{l}^{s}}{P_{1}^{s}}-\left(\frac{T_{s}}{E}\right)_{l} \frac{\delta P_{2}^{s}}{P_{2}^{s}}\right]
\end{aligned}
$$




\section{6}

ANDERSON AND ARCHAMBEAU

If an attempt is made to solve for the unknown dissipation parameters $\delta P_{1}^{*}$ and $\delta P_{2}^{*}$ from a set of $N$ measured values of $\delta \lambda_{l} / \lambda_{l}$, it is immediately clear that the system of equations so generated is indeterminate, since the coefficient matrix, composed of the energy ratios, is singular. (The sum of a row of the matrix is

$$
2 \sum_{s=1}^{N}\left[\left(\frac{V_{s}}{E}\right)_{l}-\left(\frac{T_{s}}{E}\right)_{l}\right]=0
$$

since $T_{l}=V_{l}$. This comment is equally valid for inversion of dispersion data.) However, since dissipation due to inertial effects is unlikely in the earth, at least to any appreciable degree, it seems quite reasonable to assume all the $\delta P_{\mathrm{a}} \mathrm{E} \equiv 0$. In this case all the dissipation is assigned to anelastic effects and

$$
\frac{\delta \lambda_{l}}{\lambda_{l}}=2 \sum_{s=1}^{N}\left(\frac{V_{s}}{E}\right)_{l} \frac{\delta P_{1}^{s}}{P_{1}^{s}}
$$

so that, with $M \geq N$ measured values of $\delta \lambda_{b} / \lambda_{l}$, we can formally invert the system. A leastsquares approach then gives, for example,

$$
Y=\left(A^{T} A\right)^{-1} A^{T} X
$$

where

$$
\begin{array}{ll}
Y=\delta P_{1}^{s} / P_{1}^{s} & s=1,2, \cdots, N \\
X=\delta \lambda_{l} / \lambda_{l} & l=1,2, \cdots, M \geq N \\
A=\left(V_{s} / E\right)_{l} & s=1,2, \cdots, N \\
& l=1,2, \cdots, M \geq N
\end{array}
$$

These considerations have served to indicate a systematic approach to the reduction and interpretation of measurements of dissipation for elastic waves. It remains to recast these results in more familiar terms and to verify that the abstract formulation does indeed apply to the problems of interest.

In particular, the equations of motion for the toroidal oscillations of a spherical layered earth are [Gilbert and MacDonald, 1960]

$$
\begin{aligned}
& \mu_{s} \frac{d}{d r}\left[r^{2} \frac{d}{d r} W_{l}^{(s)}\right] \\
& -\mu_{s}\left[l(l+1) W_{l}^{(s)}\right]=-\rho_{s} \omega_{l}{ }^{2} r^{2} W_{l}^{(s)}
\end{aligned}
$$

Thus the parameters previously introduced are $P_{1}^{*}=\mu_{s}, P_{2}^{*}=\rho_{s}, P_{3} \equiv-l(l+1) \equiv-(k a)^{2}$, $\lambda_{l}=\omega_{l}{ }^{2}$, and $r_{1}=r_{2}=r^{2}$. Here the wave num- ber $k$ is introduced for convenience. In the present context $a$ denotes the radius of the earth.

It is easy to show that the equation

$$
\sum_{s=1}^{N}\left\{H_{s} u_{l}^{s}, u_{l}^{s}\right\}=\lambda_{l} \sum_{s=1}^{N}\left\{P_{2}^{s} r_{2} u_{l}^{s}, u_{l}^{s}\right\}
$$

becomes

$$
\begin{gathered}
\sum_{s=1}^{N} \int_{r_{s-1}}^{r_{s}} \mu_{s}\left[\left(\frac{d W_{l}^{s}}{d r}-\frac{W_{l}^{s}}{r}\right)^{2}\right. \\
\left.+(l-1)(l+2)\left(\frac{W_{l}^{s}}{r}\right)^{2}\right] r^{2} d r \\
=\omega_{l}{ }^{2} \sum_{s=1}^{N} \int_{r s-1}^{r s} \rho_{s}\left[W_{l}{ }^{2}\right]^{2} r^{2} d r
\end{gathered}
$$

and, from first principles, that the left side of the equation is the total potential energy and the right side the total kinetic energy. We now introduce $\omega_{l}^{*} \equiv \delta \omega_{l}$ and $k_{l}^{*}=\delta k_{1}$ for the imaginary parts of the frequency and wave number respectively, and $\rho^{*}$ and $\mu^{*}$ for the imaginary parts of the density and rigidity. The partial variations of (16) take the form

$$
\frac{\mu_{s}}{\omega_{l}}\left(\frac{\omega_{l}^{*}}{\mu_{s}^{*}}\right)_{0}=\left(\frac{V_{s}}{E}\right)_{l} \quad \frac{\rho_{s}}{\omega_{l}}\left(\frac{\omega_{l}^{*}}{\rho_{s}^{*}}\right)_{0}=-\left(\frac{T_{s}}{E}\right)_{l}
$$

The dissipation function $Q^{-1}$ is defined in terms of the real and imaginary parts of the angular frequency by

$$
Q_{l}^{-1}=2 \omega_{l}^{*} / \omega_{l}
$$

and therefore, from the expressions for the total variation in the eigenvalue,

$$
Q_{l}^{-1}=2 \sum_{s=1}^{N}\left[\left(\frac{V_{s}}{E}\right)_{l}\left(\frac{\mu_{s}^{*}}{\mu_{s}}\right)-\left(\frac{T_{s}}{E}\right)_{l}\left(\frac{\rho_{s}^{*}}{\rho_{s}}\right)\right]
$$

As previously discussed, the $\rho_{s}^{*}$ are ignored as sources of dissipation, so that in general only anelastic phenomena are treated, and so

$$
Q_{l}^{-1}=\sum_{s=1}^{N}\left(\frac{V_{s}}{V}\right)\left(\frac{\mu_{s}^{*}}{\mu_{s}}\right)
$$

In this case the result agrees with (2), a result obtained by an elementary consideration of the definition of $Q^{-1}$. In (22), then, the ratio $\left(\mu_{s}^{*} / \mu_{s}\right)$ corresponds to the intrinsic $Q_{s}^{-1}$ of the layer. Thus the previous considerations leading to (2) 
are justified so long as only anelastic effects are important.

On the other hand, if

$$
I_{l}{ }^{s} \equiv\left\{u_{l}{ }^{2}, u_{l}{ }^{8}\right\}=\int_{r_{s-1}}^{r_{0}}\left[W_{l}^{s}\right]^{2} d r
$$

(15) becomes

$$
\left(\frac{1}{\omega_{l}}\right)\left(\frac{\delta \omega_{l}}{\delta k_{l}}\right)_{0}=\left(k_{l}\right) \sum_{s=1}^{N} \mu_{s} I_{l}^{s} / E
$$

Since, by definition, the density and rigidity parameters are not varied and are therefore real, $\delta \omega_{l}$ and $\delta k_{l}$ are also real. Since the group and phase velocity are defined as

$$
\begin{aligned}
U_{l} & =\left(\delta \omega_{l} / \delta k_{l}\right)_{0} \\
c_{l} & =\omega_{l} / k_{l}
\end{aligned}
$$

we have

$$
U_{l}=\left(\frac{\delta \omega_{l}}{\delta k_{l}}\right)_{0}=2 c_{l}^{-1}\left(E / \omega^{2}\right)^{-1} \sum_{s=1}^{N} \mu_{s} I_{l}^{s}
$$

Finally, it is possible to show how measured values of $k_{l} * / k_{l}$, corresponding to measurements of the attenuation of a propagating wave, are connected with measurements of $\omega_{l}^{*} / \omega_{l}$. The latter corresponds to measurements of the decrement in time of a standing wave, or $Q^{-1}$ as usually defined.

Thus from (13), constraining $\delta \lambda_{l}$ to vanish, partials may be formed under the conditions that $\delta P_{j}{ }^{r}$ vanish except the one $\delta P_{i}{ }^{s}$. Therefore

$$
\left(\frac{\delta P_{3}}{\delta P_{i}^{s}}\right)_{0}=\left\{H_{i}{ }^{s} u_{l}{ }^{8}, u_{l}{ }^{s}\right\} / \sum_{s=1}^{N}\left\{P_{1}{ }^{s} u_{l}{ }^{s}, u_{l}{ }^{s}\right\}
$$

Thus, for the application under consideration, this becomes, for the anelastic effects,

$$
\frac{\mu_{s}}{k_{l}}\left(\frac{k_{l}^{*}}{\mu_{s}^{*}}\right)_{0}=V_{s} / k_{l}{ }^{2} \sum_{r=1}^{N} \mu_{r} I_{l}^{r}
$$

The result of (26) gives, finally,

$$
\frac{\mu_{s}}{k_{l}}\left(\frac{k_{l}^{*}}{\mu_{s}^{*}}\right)_{0}=\frac{c}{U}\left(\frac{V_{s}}{E}\right)
$$

From (20) and (21), therefore,

$$
Q_{l}^{-1}=\frac{2 \omega_{l}^{*}}{\omega_{l}}=\left(\frac{U}{c}\right) \frac{2 k_{l}^{*}}{k_{l}}
$$

which gives the relationship between the two types of measurement. These results are quite clearly valid for any type of elastic wave.

Numerical calculations. Detailed tables and graphs of the functions $\mu \delta c / \delta \mu$ and $\rho \delta c / \delta \rho$ have been presented elsewhere [Anderson, 1963, 1964]. These tables and graphs were prepared for the interpretation of phase velocity data to find $\rho$ and $\mu$. By writing

$$
\begin{aligned}
\mu \delta c / \delta u & =-c\left(\mu / \mu^{*}\right)\left(k^{*} / k\right) \\
& =-(c / 2)\left(Q^{-1} / Q_{\beta}{ }^{-1}\right)_{\rho} \\
\rho \delta c / \delta \rho & =+c\left(\rho / \rho^{*}\right)\left(k^{*} / k\right) \\
& =+(c / 2)\left(Q^{-1} / Q_{\rho}^{-1}\right)_{\mu}
\end{aligned}
$$

where $Q_{\beta}{ }^{-1}$ and $Q_{\rho}^{-1}$ are the contributions to $Q_{0}{ }^{-1}$ caused by imperfect elasticity and imperfect inertia, we may use these calculations for the problem at hand. Figure 1 is a plot of the function $-(c / 2)\left(Q^{-1} / Q_{\beta}{ }^{-1}\right)_{0}$ versus period for some of the layers in the CIT13F oceanic model. This is the standard earth model upon which the Universal Dispersion Tables [Anderson, 1964] are based and which satisfies long-period oceanic Love wave and torsional oscillation data. The calculations were performed using the earth-stretching approximation [Anderson and Toksöz, 1963].

Each member of this family of curves indicates the effect of anelastic dissipation in the corresponding layer on observed Love wave and torsional oscillation amplitudes. For example, if a dissipative layer is present at a depth of 186.5 $\mathrm{km}$ in an otherwise perfectly elastic earth, there will be a peak in the observed Love wave $Q^{-7}$ spectrum centered at about 250 sec. Interpretation of observed values of $Q$ requires finding a linear combination of these curves that matches the experimental data. The uniqueness problem is identical to that in dispersion interpretations.

The amplitudes of these curves are proportional to layer thickness and may be scaled to other thicknesses as appropriate.

Data analysis. $Q$ has been determined from free oscillation data by Smith [1961], Alsop et al. [1961], MacDonald and Ness [1961], and Connes et al. [1962]. Attenuation of longperiod surface waves from which $Q$ can be determined has been measured by Ewing and Press [1954a, b], Satô [1958], Popov [1960], Bäth and López Arroyo [1962], Press et al. [1961], Arkhangel'skaya and Fedorov [1961], Savarensky et al. [1961], and Ben-Menahem and Tok- 


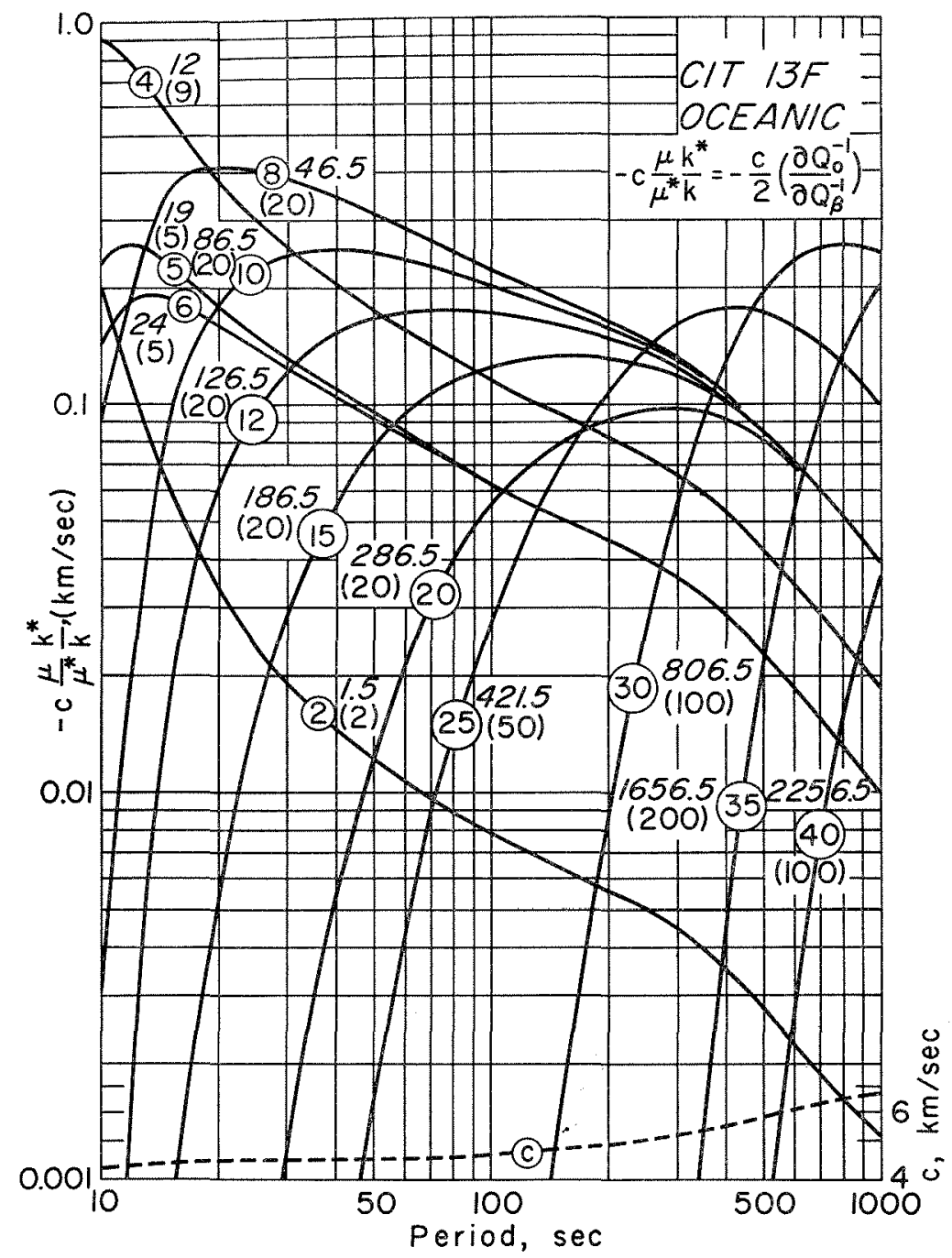

Fig. 1. Curves showing the $\delta Q_{o}^{-1}$ for Love waves or torsional oscillations resulting from a $\delta Q_{\beta}{ }^{-1}$ in various layers of the CIT13F oceanic earth model. If the starting trial structure is perfectly elastic $\delta Q^{-1}=Q^{-1}$. The $\delta Q_{\beta}^{-1}$ are due to complex rigidities.

söz [1963 and personal communication]. Shorterperiod data have been obtained by Gutenberg and Richter [1935] and Wilson [1940], and the present authors have obtained several points from the Naña strain record of the Chilean earthquake as a preliminary to the Fourier analysis of this record. Since there are so few data no attempt was made to segregate them according to path or author in presenting them in Figure 2. A detailed discussion of the data is being prepared in conjunction with the experimental study to follow. For present purposes we accept the data uncritically, realizing that effects other than anelasticity contribute to the observations.

The data show appreciable scatter, but several trends are evident: (1) The free oscillation data reveal a $Q$ consistently greater than the shorter-period surface wave data. (2) For periods greater than some 100 to 200 seconds $Q$ increases rapidly with increasing period. (3) There is some evidence for an increase of $Q$ for periods less than about $40 \mathrm{sec}$, although there are few data here. (4) $Q$ seems to have a broad 


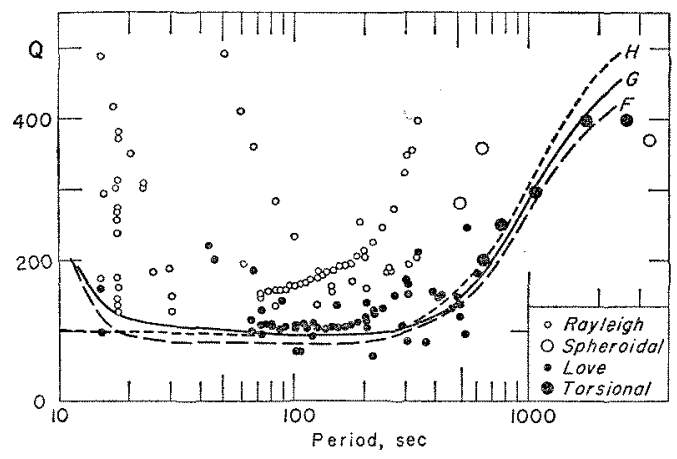

Fig. 2. Love wave, Rayleigh wave, spheroidal oscillation, and torsional oscillation data for the earth as a function of period. Measurements of traveling wave attenuation have been converted to $Q$ by equation 29 . The curves are theoretical results discussed in the text.

minimum centered near 100 sec. At this point the waves are sampling well into the mantle. (5) $Q$ for Rayleigh waves is consistently greater than $Q$ for Love waves, a result that is not surprising since Rayleigh waves involve compression as well as shear. (6) The minimum value of $Q$ for Love waves, about 100 , is significantly less than measured under laboratory conditions for such rocks as granite and dunite. This is a significant point since the elastic properties of the mantle, even in the low-velocity zone, are generally greater than those measured in the laboratory.

The variation of $Q$ with period we attribute to its variation with depth under the assumption that $Q$ for the material of the earth is intrinsically frequency independent. This is the opposite approach from that taken by those authors who combine long-period measurements with body wave measurements to support a given $Q$ frequency law [Lomnitz, 1962] or even to support a frequency-independent $Q$ law [Knopoff and MacDonald, 1958]. Since different wavelengths sample to different depths in the earth and since $Q$ is so sensitive to temperature, pressure, and the compositional and phase changes which mask any intrinsic frequency dependence, we maintain that the latter approach is not valid, at least at this stage of development. Much better data may eventually allow the extraction of a frequency-dependent term.

Before attempting to find the detailed distribution of $Q$ in the mantle, we investigate the following simpler problems:
1. If the rapid increase in $Q$ at long periods is due to a discontinuous increase in $Q$ at some depth in the mantle, what is the most probable depth of this discontinuity?

2. If a minimum in $Q$ exists and is due to the insertion of a low $-Q$ layer in an otherwise high- $Q$ earth, what can we tell about the properties of this layer?

3. If the implied variation of $Q$ with depth is due to the effects of temperature and pressure alone, what are the corresponding thermodynamic properties, can we place bounds on them, and what do they tell us about possible mechanisms of dissipation? This problem will be discussed at length in later publications.

Of course the actual variation is due to the combined effects of temperature and pressure and heterogeneity in phase and composition, but it is instructive at this point to investigate the effects separately.

To develop an intuition for the appropriate numbers to be used in these numerical experiments Anderson and Kovach [1964] analyzed the attenuation of multiple $S c S$ and $s S c S$ phases from a deep-focus earthquake in South America. They were able to estimate average values for $Q$ for the region above and below the depth of focus. Above $600 \mathrm{~km}$ the average $Q$ was determined to be about 180 . The lower mantle had an average $Q$ of at least 1400 .

In the first experiment, we assumed that the upper mantle had a constant $Q$ of 200 and that

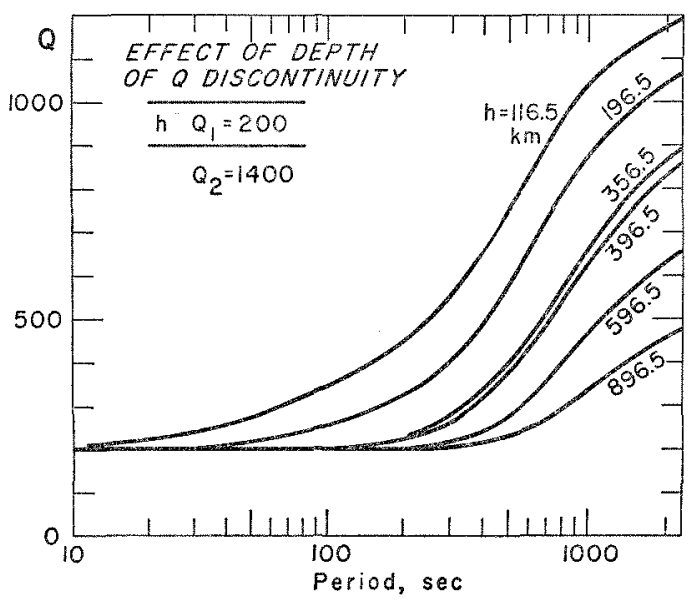

Fig. 3. Numerical experiments to investigate the effect of depth on $a Q$ discontinuity where the $Q$ contrast is constant $(200 / 1400)$. 


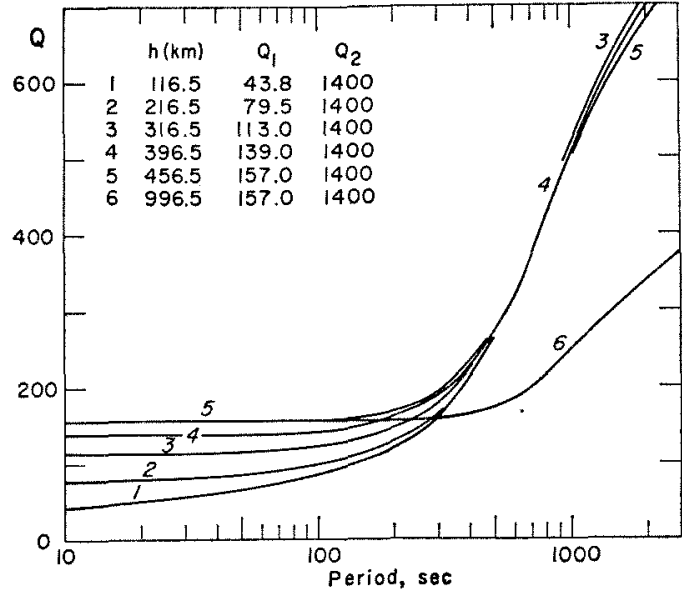

Fig. 4. Numerical experiments to investigate the effect of depth of a $Q$ discontinuity where the average $Q$ in the upper $600 \mathrm{~km}$ is kept at 200 , except for curve 6 where the $Q$ is 157 .

the lower mantle had a constant $Q$ of 1400 . We investigated the variation of $Q$ for Love waves that would result from placing the interface at different depths. Figure 3 gives the results. The rapid increase of $Q$ with period for the Love wave data (see Figure 2) begins between about 150 and 300 sec. Figure 3 indicates that the corresponding discontinuity is somewhere between about 350 and about $600 \mathrm{~km}$ deep. These numbers are tantalizingly close to the depth of the much-discussed inhomogeneous $C$ region, of the mantle, and to depths of supposed phase changes.

The large uncertainty in the assigned depth is due partially to the sparseness and scatter of data in the period range between 200 and 500 sec which makes it difficult to determine at what period the low values associated with mantle waves rise to meet the higher values associated with the free oscillations. This is the period range where both free oscillation and surface wave experiments are difficult to perform. We are now filling this experimental gap, since it is crucial in discussions of the location and properties of phase changes in the upper mantle. Even now, however, we can say that if a $Q$ discontinuity exists it is certainly deeper than 200 $\mathrm{km}$ and therefore more likely to be associated with a feature similar to Jeff reys' original concept of the ' $20^{\circ}$ discontinuity' than to Gutenberg's shallower low-velocity zone. Recent sur- face wave interpretations [Anderson and Toksöz, 1963; Anderson, 1964], however, have pushed the low-velocity zone down as deep as 350 to $500 \mathrm{~km}$, which effectively makes the bottom of the low-velocity zone the top of the inhomogeneous $C$ region and links these two controversial features.

We then varied the depth of the interface and at the same time varied the $Q$ of the upper layer so as to maintain an average $Q$ of 200 in the upper $600 \mathrm{~km}$. These results are shown in Figure 4. In this experiment a depth of $450 \mathrm{~km}$ or deeper is suggested, strengthening the arguments in the preceding paragraph.

The single-interface model, of eourse, is much too simple a picture even though it does indicate the depth where a rapid increase is most likely. A discontinuous increase in $Q$ to 1400 , the average for the lower mantle, leads to a more rapid variation with period than is observed. This is evidence for an increase of $Q$ with depth in the deeper mantle, and it indicates a value appreciably less than 1400 immediately below the discontinuity, increasing to a value somewhat greater than 1400 in the bottom of the mantle, if the value 1400 is an appropriate average. An increase of $Q$ at shorter periods also cannot be explained by a simple one-discontinuity model. A minimum in a $Q$ versus $T$ plot would suggest that there is a low- $Q$ layer in the upper mantle which does not rise to the surface.

Let us consider, therefore, the second question. We will insert a layer of anomalously low $Q$ into a previously constant- $Q$ earth model. The data will then be interpreted as the depar-

TABLE 1. Properties of Anomalous $Q$ Layers Inserted at Various Depths Which Give an Observed Minimum $Q$ of 100 at $T_{m}$. If the Background $Q$ is 200

\begin{tabular}{|c|c|c|c|c|c|}
\hline Layer & $\begin{array}{l}T_{m} \\
\mathrm{sec}\end{array}$ & $\begin{array}{l}\text { Depth, } \\
\text { km }\end{array}$ & $\begin{array}{c}\text { Thickness, } \\
\text { km }\end{array}$ & $Q_{\beta}$ & $Q_{600}$ \\
\hline 12 & 78 & 126.5 & $\begin{array}{r}20 \\
50 \\
100 \\
200\end{array}$ & $\begin{array}{l}14 \\
32 \\
55 \\
87\end{array}$ & 150 \\
\hline 15 & 160 & 186.5 & $\begin{array}{r}20 \\
100 \\
200\end{array}$ & $\begin{array}{l}10 \\
44 \\
72\end{array}$ & 141 \\
\hline 20 & 285 & 286.5 & $\begin{array}{r}20 \\
100 \\
200 \\
300\end{array}$ & $\begin{array}{r}7 \\
32 \\
55 \\
73\end{array}$ & 125 \\
\hline
\end{tabular}


TABLE 2. Layer Thicknesses, $H$, and Dissipation Functions, $Q$, for Models A through $\mathrm{H}$

\begin{tabular}{rrrrrrrrrrr}
\hline$H, \mathrm{~km}$ & $Q(\mathrm{~A})$ & $Q(\mathrm{~B})$ & $Q(\mathrm{C})$ & $H, \mathrm{~km}$ & $Q(\mathrm{D})$ & $Q(\mathrm{E})$ & $H, \mathrm{~km}$ & $Q(\mathrm{~F})$ & $Q(\mathrm{G})$ & $Q(\mathrm{H})$ \\
\hline 7.5 & 200 & 225 & 150 & 7.5 & 200 & 200 & 56.5 & 200 & 200 & 100 \\
29.0 & 200 & 330 & 220 & 69.0 & 100 & 100 & 100.0 & 60 & 80 & 100 \\
40.0 & 100 & 105 & 70 & 80.0 & 80 & 80 & 240.0 & 80 & 80 & 80 \\
60.0 & 40 & 60 & 40 & 160.0 & 120 & 200 & 50.0 & 100 & 100 & 200 \\
80.0 & 150 & 180 & 120 & 480.0 & 300 & 500 & 250.0 & 300 & 300 & 400 \\
140.0 & 200 & 200 & 200 & 1150.0 & 1400 & 1500 & 300.0 & 800 & 900 & 800 \\
440.0 & 600 & 800 & 800 & 953.0 & 2000 & 2000 & 1900.0 & 1600 & 1700 & 2000 \\
400.0 & 800 & 900 & 1000 & & & & & & & \\
400.0 & 1000 & 1200 & 1500 & & & & & & & \\
1300.0 & 1400 & 1600 & 2000 & & & & & & &
\end{tabular}

ture from the background $Q$ caused by the anomalous layer.

If the $Q$ anomaly is $\Delta Q_{\beta}^{-1}$ and the observed anomaly is $\Delta Q_{0}{ }^{-1}$,

$$
\Delta Q_{0}^{-1} / \Delta Q_{\beta}^{-1}=\delta Q_{0}^{-1} / \delta Q_{\beta}^{-1}
$$

This ratio can be obtained from the curves in Figure 1. If the background $Q$ is 200 and the observed minimum $Q$ is 100 , we can determine the $Q$ of the anomalous layer, given its depth and thickness. Results for three different assigned depths are given in Table 1. For example, if a $200-\mathrm{km}$-thick anomalous layer is centered at a depth of $286.5 \mathrm{~km}$ and a $Q$ of 100 is observed at $285 \mathrm{sec}$, the $Q$ of the layer is 55 and the $Q$ of the surrounding material is 200 . The values in this table are determined directly from Figure 1. Also given are the values for $Q$ which would be observed at 600 sec, assuming a background $Q$ of 200 . The fact that the $Q$ at 600 sec, in all cases, is less than that observed indicates that the 'background' $Q$ at depth is greater than assumed and again suggests a $Q$ increasing with depth in the deeper mantle and/or a discontinuous increase in $Q$ somewhere in the upper mantle.

The third simplest assumption is that the earth is homogeneous and the observed variation of $Q$ is due only to the effects of temperature and pressure. We assume that the dissipation obeys an equation of the type

$$
\begin{aligned}
& Q^{-1}(T, P) \\
& \quad=Q_{0}{ }^{-1} \exp E^{*}\left(T_{0}-T\right) /\left(k T_{0} T\right) \\
& \quad \cdot \exp p \Delta V^{*} /(k T)
\end{aligned}
$$

where $E^{*}$ and $\Delta V^{*}$ are the activation energy and the activation volume appropriate for the mechanism. We assume that only one mechanism is operative throughout the mantle and, further, that we are far from any natural resonant frequency. We have evaluated (31) for a variety of parameters, using the pressuredepth relation of Bullard [1957] and the temperature-depth relation of Tozer [1959]. The more interesting results are shown in Figure 5, plotted step-wise for easier comparison with later figures, and summarized in Table 3. Ap-

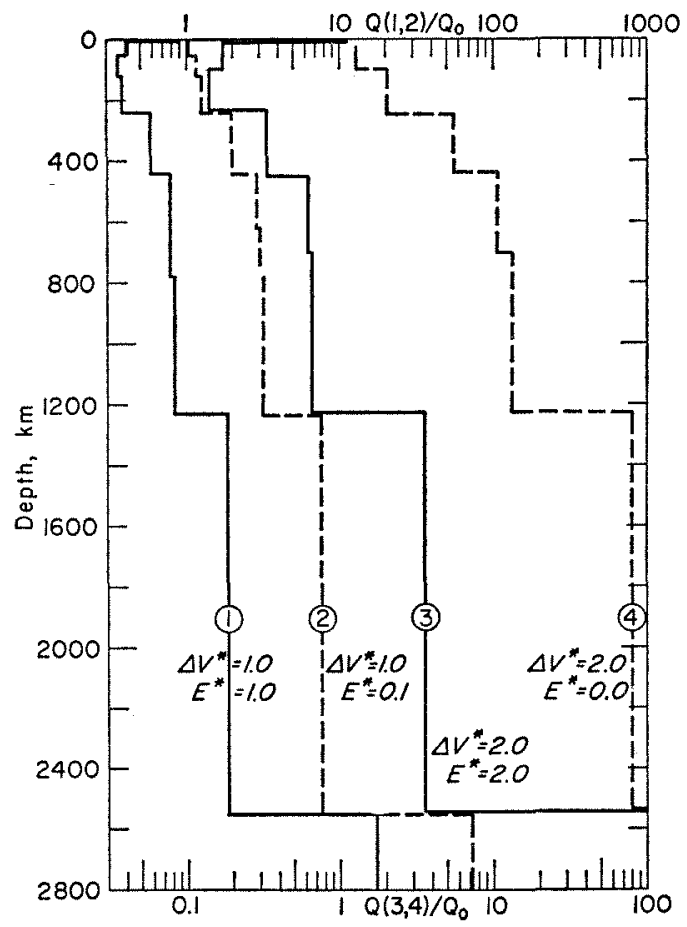

Fig. 5. The combined effects of temperature and pressure on $Q$ in the earth for various assumed activation volumes and activation energies. 
TABLE 3. Variation of $Q^{-1}(T, P)=Q^{-1}(z)$ with Depth $\left(Q_{0}^{-1}\right.$ is surface value)

\begin{tabular}{|c|c|c|c|c|c|c|c|}
\hline & \multirow{2}{*}{$\begin{array}{c}\Delta V^{*} \\
\mathrm{~cm}^{3} / \text { mole }\end{array}$} & \multicolumn{6}{|c|}{ Depth $z, \mathrm{~km}$} \\
\hline & & 0 & 26 & 100 & 340 & 540 & 1600 \\
\hline \multicolumn{8}{|c|}{$\begin{array}{c}E^{*}=0.1 \mathrm{kcal} / \mathrm{mole} \\
Q^{-1}(z) / Q_{0}^{-1}\end{array}$} \\
\hline & 0.1 & 1.00 & 0.92 & 0.91 & 0.95 & 0.98 & 1.07 \\
\hline & 0.2 & 1.00 & 0.93 & 0.94 & 1.03 & 1.10 & 1.33 \\
\hline & 1.0 & 1.00 & 1.03 & 1.18 & 2.00 & 2.82 & 7.60 \\
\hline \multicolumn{8}{|c|}{$\begin{array}{c}E^{*}=0.2 \mathrm{kcal} / \mathrm{mole} \\
Q^{-1}(z) / Q_{0}^{-1}\end{array}$} \\
\hline & 0.1 & 1.00 & 0.83 & 0.79 & 0.82 & 0.85 & 0.90 \\
\hline & 0.2 & 1.00 & 0.84 & 0.82 & 0.90 & 0.95 & 1.14 \\
\hline & 1.0 & 1.00 & 0.93 & 1.02 & 1.74 & 2.44 & 6.50 \\
\hline \multicolumn{8}{|c|}{$\begin{array}{c}E^{*}=1.0 \mathrm{kcal} / \mathrm{mole} \\
Q^{-1}(z) / Q_{0}^{-1}\end{array}$} \\
\hline & 0.1 & 1.00 & 0.37 & 0.28 & 0.28 & 0.27 & 0.27 \\
\hline & 0.2 & 1.00 & 0.37 & 0.29 & 0.30 & 0.30 & 0.33 \\
\hline & 1.0 & 1.00 & 0.42 & 0.36 & 0.58 & 0.78 & 1.90 \\
\hline \multicolumn{8}{|c|}{$\begin{array}{c}E^{*}=2.0 \mathrm{kcal} / \mathrm{mole} \\
Q^{-1}(z) / Q_{0}^{-1}\end{array}$} \\
\hline & 2.0 & 1.00 & 0.17 & & 0.34 & 0.61 & 3.58 \\
\hline & 5.0 & 1.00 & 0.25 & & 4.03 & 20.9 & 2480 \\
\hline \multicolumn{8}{|c|}{$\begin{array}{c}E^{*}=5.0 \mathrm{kcal} / \mathrm{mole} \\
Q^{-1}(z) / Q_{0}^{-1}\end{array}$} \\
\hline & 2.0 & 1.00 & 0.008 & & 0.005 & 0.008 & 0.035 \\
\hline & 5.0 & 1.00 & 0.01 & & 0.07 & 0.29 & 24.2 \\
\hline
\end{tabular}

parently there is little difficulty in fitting almost any desired depth distribution of $Q$ with reasonable assumptions concerning the parameters. Except for the upper mantle, we have probably overestimated the effect of temperature since the proper reference temperature will be an increasing function of pressure. The values in Table 3 and an additional set of calculations not presented here suggest that an appropriate activation volume is a very small fraction of the molecular volume.

Detailed models. We now attempt to determine a $Q$-versus-depth function that satisfies the Love wave and torsional oscillation data. The layer potential energy ratios used in the inversion are computed routinely by the IBM 7090 program described by Anderson and Toksöz [1963]. The following results are derived from the CIT11A oceanic model. This model has rapid changes in elastic properties which set in at about 360 and $660 \mathrm{~km}$ and which result in shear wave travel-time discontinuities at about $20^{\circ}$ and $25^{\circ}$, respectively. The quality of the present data does not justify a detailed analysis. Six or seven layers are sufficient to approximate the variation of $Q$ with depth for present purposes. The data must be more precise and cover a larger range before more detailed $Q$ structures are justified.

Even so, a large number of models have been investigated in order to exploit the sensitivity of the method. Results for some of the more interesting models are shown in Figures 2 and 6. The corresponding $Q$ models are shown in Figure 7. Results of serious attempts to fit the Love wave and torsional oscillation data are shown in Figure 2. The corresponding models are all very similar, characterized by a broad low- $Q$ layer terminated by a rapid increase in $Q$ at a depth of about $400 \mathrm{~km}$. The determination of the details of the transition region must await better data, but the present data are satisfied by a rapid increase in $Q$ between 400 and $1000 \mathrm{~km}$ followed by a constant- $Q$ lower 


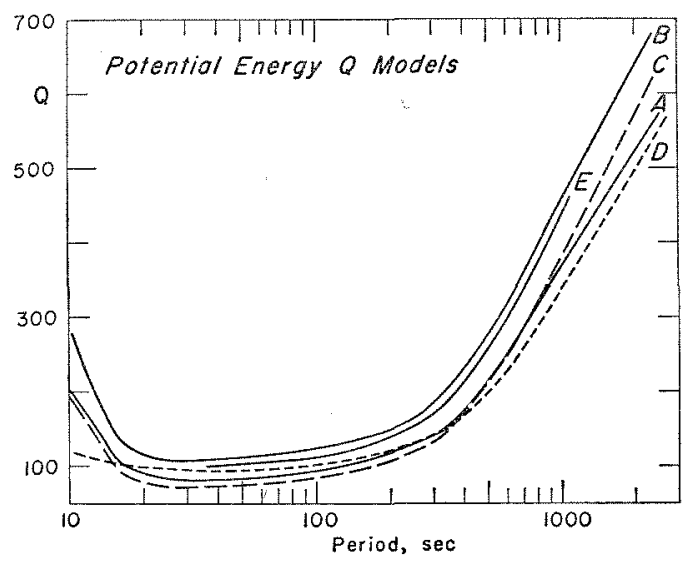

Fig. 6. Love wave $Q$ versus period for several models of $Q$ versus depth.

mantle. The details of the distribution of $Q$ in the upper $100 \mathrm{~km}$ are not reliable because of the lack of short-period data. However a value of $Q$ of approximately 80 to 100 in the lowvelocity region of the CIT11A model seems to be supported by the data. An average $Q$ of

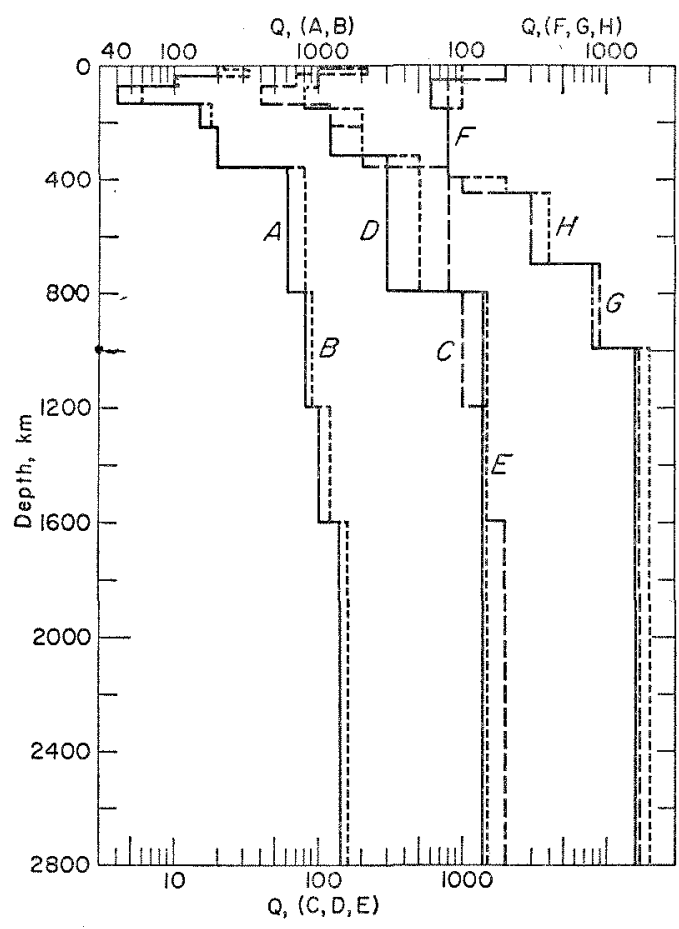

Fig. 7. Variation of $Q$ with depth for models $\mathrm{A}$ through $\mathrm{H}$. Models $\mathrm{F}, \mathrm{G}$, and $\mathrm{H}$ are the most satisfactory (see Figure 2). about 400 in the transition region (400 to 1000 $\mathrm{km}$ ) seems to be indicated. An increase in $Q$ by at least an order of magnitude between 400 and $1000 \mathrm{~km}$ seems to be a required conclusion. Because of the great sensitivity of $Q$ to phase changes, it is tempting to accept this as good evidence for a phase change in the upper mantle. We prefer to postpone discussion of this point to a later paper in which the anelastic and elastic properties will both be brought to bear on the subject.

The $Q$ models presented in this paper represent first approximations to the actual $Q$ structure of the earth and should be regarded as only tentative. In particular, no attempt has been made to force agreement with the body wave data of Anderson and Kovach [1964], although this additional constraint can be satisfied by small changes in the tabulated models without violating any of the major conclusions. An average $Q$ of about 2000 in the lower $1000 \mathrm{~km}$ of the mantle and a slightly higher $Q$ in the upper $600 \mathrm{~km}$ than are given by models $\mathrm{F}, \mathrm{G}$, and $H$ will improve the fit to the surface wave data and also satisfy the $S c S$ data. There is no need to invoke a frequency-dependent $Q$ in order to satify the available data. (See Table 2.)

Acknowledgments. The research was supported by AF-AFOSR-25-63 of the Air Force Office of Scientific Research as part of the Advanced Projects Agency project Vela.

We wish to acknowledge helpful discussions with Stewart Smith and David Harkrider. Ari BenMenahem and M. N. Toksöz permitted us to use some of their unpublished data. Freeman Gilbert and George Backus read the manuscript and made many helpful suggestions.

\section{REFERENCES}

Alsop, L., G. H. Sutton, and M. Ewing, Measurement of $Q$ for very long period free oscillations, J. Geophys. Res., 66, 2911-2915, 1961.

Anderson, Don L., Recent evidence concerning the structure and composition of the earth's mantle, Physics and Chemistry of the Earth, chap. 1, 1-129, Pergamon Press, New York and London, 1963.

Anderson, Don L., Universal dispersion tables, 1, Love waves across oceans and continents, in press, Bull. Seismol. Soc. Am, 1964. (Also available as Seismol. Lab. Tech. Rept. 42, 1963.)

Anderson, Don L., and R. L. Kovach, Attenuation in the mantle and rigidity of the core from multiply reflected core phases, Proc. Natl. Acad. Sci. U. S., 51, 168-172, 1964.

Anderson, Don L., and M. N. Toksöz, Surface 
waves on a spherical earth, 1, Upper mantle structure from Love waves, J. Geophys. Res., 68, 3483-3500, 1963.

Anderson, Don L., M. N. Toksöz, and R. Kovach, Upper mantle structure from long period surface waves, Intern. Union Geod. and Geophys., XIII General Assembly, Berkeley, 1963.

Archambeau, C., and Don L. Anderson, Inversion of surface wave dispersion data, Intern. Union Geod. and Geophys., XIII General Assembly, Berkeley, 1963.

Arkhangel'skaya, V. M., and S. A. Fedorov, Damping of surface Rayleigh waves, Izv. Akad. Nauk SSSR, Ser. Geofiz., 738-743, 1961.

Båth, M., and A. López Arroyo, Attenuation and dispersion of $G$ waves, J. Geophys. Res., 67, 1933-1942, 1962.

Ben-Menahem, A., and M. N. Toksöz, Sourcemechanism from spectra of long-period surface waves, 2, The Kamchatka earthquake of November 4, 1952, Seismol. Lab. Tech. Rept. 29, May 1963.

Bullard, E. C., The density within the earth, Verhandel Ned. Geol. Mijnbouwk. Genoot, Geol. Ser., 18, 23-41, 1957.

Connes, J., P. A. Blum, G. and N. Jobert, Observation des oscillations propres de la terre, Ann. Geophys., 18, 260-268, 1962.

Ewing, M., and F. Press, An investigation of mantle Rayleigh waves, Bull. Seismol. Soc. Am., 44, $127-147,1954 a$.

Ewing, M., and F. Press, Mantle Rayleigh waves from the Kamachatka earthquake of November 4, 1952, Bull. Seismol. Soc. Am., 44, 471-479, $1954 b$.

Fortsch, O., Ableitung des von der Frequenz unabhangigen Absorbtionsk-oeffizienten aus Maschinenschwingungen, $Z$. Geophys., 16, 1-2, 1940.

Gilbert, F., and G. J. F. MacDonald, Free oscillations of the earth, 1, Toroidal oscillations, $J$. Geophys. Res., 65, 675-693, 1960.

Gutenberg, B., and C. F. Richter, On seismic waves, 2, Gerlands Beitr. Geophys., 45, 280-360, 1935.
Jeffreys, H., Small corrections in the theory of surface waves, Geophys. J., 6, 115-117, 1961.

Knopoff, L., and G. J. F. MacDonald, Attenuation of small amplitude stress waves in solids, Rev. Mod. Phys., 30, 1178-1192, 1958.

Lomnitz, C., Application of the logarithmic creep law to stress wave attenuation in the solid earth, J. Geophys. Res., 67, 365-368, 1962.

MacDonald, G. J. F, and N. Ness, A study of the free oscillations of the earth, $J$. Geophys. Res., $66, \mathbf{1 8 6 5 - 1 9 1 2 , 1 9 6 1 .}$

Meissner, E., Elastische oberflachen Querwellen, Verhandl. Intern. Kongr. Tech. Mech., Znd, Zurich, 3-11, 1926.

Ness, N., J. C. Harrison, and L. B. Slichter, Observations of the free oscillations of the earth, $J$. Geophys. Res., 66, 621-629, 1961.

Popov, I. I., Dispersion of long-period Love waves in the continental and oceanic crust along the path Indonesia-Crimea, Izv. Akad. Nauk SSSR, Ser. Geofiz, 10, 1960.

Press, F., A. Ben-Menahem, and M. N. Toksöz, Experimental determination of earthquake fault length and rupture velocity, J. Geophys. Res., $66,3471-3485,1961$.

Savarensky, E. F., I. I. Popov, and A. P. Lazareva, Observations of long periods waves of the Chilean earthquake of 1960, Izv. Akad. Nauk SSSR, Ser. Geofiz, 744-748, 1961.

Satô, $Y$, Attenuation, dispersion and the wave guide of the $G$ wave, Bull. Seismol. Soc. Am., 48, 231-251, 1958.

Smith, S., An investigation of the earth's free oscillations, Thesis, California Institute of Technology, Pasadena, 1961 (also personal communication).

Tozer, D. C., The electrical properties of the earth's interior, in Physics and Chemistry of the Earth, vol. 3, Pergamon Press, London, 1959.

Wilson, J. T., The Love waves of the South Atlantic earthquake of August 28, 1933, Bull. Seismol. Soc. Am., 30, 273-301, 1940.

(Manuscript received February 18, 1964.) 